\title{
Reliability assessment of pre -post test questionnaire on the impact of a daylong clinical pharmacology workshop among medical professionals
}

\author{
Arunava Biswas', Sukanta Sen², Krishnangshu Ray ${ }^{3}$ \\ ${ }^{1}$ Associate Professor, Department of Pharmacology, Coochbehar Government Medical College and Hospital, \\ Coochbehar, West Bengal,India, ${ }^{2}$ Professor and Head, Department of Pharmacology, ICARE Institute of Medical, \\ Sciences and Research, Haldia, West Bengal, India, ${ }^{3}$ Professor and Director, Institute of Health and Family Welfare, \\ Government of West Bengal, India
}

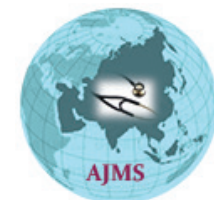

Background: Pretest-posttest questionnaire are often used during workshop to assess the impact of a program on the participants. However, the reliability of such data collection 'tool' is not often judged. There is lack of evidence supporting the authentication of such research instrument. Aims and Objective: This study was conducted to assess the reliability of prepost test questionnaire comprising MCQs, on participants attending a one-day workshop on clinical pharmacology at a teaching hospital in eastern India. Materials and Methods: Pre and post test questionnaire comprising $15 \mathrm{MCQs}$ covering different aspects of 'Good Clinical Practices' on which several presentations were later delivered by experts were distributed to $(n=42)$ participants in a one-day oriented workshop within stipulated time period and their feedback were collected and statistically analyzed after the workshop was over. Results: The average number of MCQs attempted in the pretest questionnaire was 12.42 and in posttest were 14.44 by the participants. The workshop participants increased their percentage of correct answer score from an average of 48.4 in pretest to a statistically significant 88.73 in posttest $(p<0.0001)$. Conclusion: Pre/post questionnaire could be effective and reliable tools in meeting stipulated learning objectives and help in assessing the aftermath of an educational program.

Key words: Pre-test; Post-test; Questionnaire; Workshop; Reliability
http://nepjol.info/index.php/AJMS DOI: 10.3126/ajms.v10i6.25448 E-ISSN: 2091-0576 P-ISSN: 2467-9100

\section{INTRODUCTION}

Learner's outcome of any educational program is commonly evaluated by pre/post questionnaire. Questionnaire is a predetermined set of questions used to collect data. ${ }^{1}$ It is a data collection 'tool' for collecting and recording information about a particular issue of interest. 2,3 This procedure provides feedback to the instructor by measuring the initial knowledge level of the learner and what knowledge the learner gained from the workshop or presentation. ${ }^{4}$ Ideally, a participant would not answer any question they did not know or if there is some confusion. After gaining some knowledge from an educational program a learner might attempt and give correct answers to more questions. As instructors, measuring the learners' baseline knowledge and its accountability at the beginning of any educational program helps in assessing the impact of the workshop at the end by conducting posttest. It is important to appraise the objective of a successful conduct of workshop. ${ }^{5}$

Workshops are ideally expected to be very interactive with hands on training of the participants. But unfortunately, the actual pictures of workshop remain restricted to series of didactic lectures like a one-way traffic leading to loss of interest on the subject of discussion and finally end up 
with an unsuccessful event without any long-term impact on the participants. Thus, to assess the actual impact of workshop on the participants often pre and posttest comprising of same set of numerous multiple-choice questions (MCQs) are used during workshop. A novel approach by the use of pre- and post-testing is used to take the element of guessing at answers to questions out of this method to better quantifying knowledge gained by participants in a workshop. ${ }^{1} \mathrm{MCQ}$ s are the most frequently used tool to assess cognitive skill of an individual. ${ }^{6} \mathrm{MCQs}$ help in retention of information acquired from educational sessions. ${ }^{7}$ When we think of reliability of a tool, it refers to the degree to which the results obtained by a measurement and procedure can be replicated. ${ }^{8,9}$ Thus, to prove a tool reliable we need to assess its importance with scientific research work. Reliability could be assessed by many ways; one of them is the test-retest reliability which was applied in this study. ${ }^{10}$ Since there are limited publications on this issue the study was planned to assess the reliability of preposttest questionnaire comprising MCQs on participants attending a one-day workshop on clinical pharmacology at a teaching hospital.

\section{MATERIALS AND METHODS}

A Clinical Pharmacology one day-oriented workshop was conducted in the Department of Pharmacology in a tertiary care hospital in the eastern part of India in April 2018 on 'Good Clinical Practices (GCP)'. The participants attended were faculties from various clinical, pre and para clinical departments of the institute along with members of the institutional ethics committee and few post graduate students. The participants attending the workshop were made aware of the fact that the filled-up questionnaire of pre-posttest will be handled confidentially and analyzed for scientific purpose. A written informed consent was obtained from the attendees prior to dispensation of the questionnaire. The preset questionnaire of 15MCQs covering various aspects of Good Clinical Practices and topics later covered by the speakers, was distributed to the participants $(n=42)$ at the initiation of the programme before commencement of the presentations by various experts. Each question had a single correct answer. The participants were given 5 minutes time to complete and submit the questionnaire. Attempting all the MCQs were mandatory although the participant had the right to leave in case, they were not sure of the answer. Declaration of self-identity was made optional on the questionnaire they were provided. When the daylong session was over at the end of all the presentations and interactive sessions the participants were again provided the same set of questionnaires and asked to submit within stipulated time period. Later the response of the participants was statistically analyzed and calculated with the help of software GraphPadInstat $3^{\circledR}$ calculating mean, percentage and unpaired t test. A $p$ value of $<0.05$ was considered to be statistically significant. The analysis of the study was not shared later with the participants as this was done solely as an intradepartmental academic activity to assess the impact of the workshop on the participants and explore the scope of any improvement required for better outcome of such educational programme in the future endeavors.

\section{RESULTS}

Out of total ( $n=42)$ participants, $(n=4)$ didn't complete the pre/post questionnaire as they left the programme before its completion without any intimation. The ratio of male and female participants of the workshop was 29:13. The performances of the participants are presented in Table 1.

The average number of MCQs attempted in the pretest questionnaire was 12.42 and in posttest were 14.44 by the participants. The workshop participants increased their percentage of correct answer score from an average of 48.4 in pretest to a statistically significant 88.73 in posttest $(\mathrm{p}<0.0001)$.

\section{DISCUSSION}

This pilot study was undertaken to evaluate the reliability of pre-posttest MCQs questionnaire in a daylong workshop conducted at a tertiary care hospital. The study investigated the role of such questionnaire as far as the

\begin{tabular}{|c|c|c|}
\hline \multirow[t]{2}{*}{ Question } & $\begin{array}{l}\text { Pre-test results } \\
\qquad(n=42)\end{array}$ & $\begin{array}{c}\text { Post-test } \\
\text { results }(n=38)^{*}\end{array}$ \\
\hline & $\%$ Correct & $\%$ Correct \\
\hline Q1 & 24 & 100 \\
\hline Q2 & 48 & 78 \\
\hline Q3 & 35 & 95 \\
\hline Q4 & 45 & 90 \\
\hline Q5 & 76 & 98 \\
\hline Q6 & 22 & 68 \\
\hline Q7 & 45 & 90 \\
\hline Q8 & 68 & 80 \\
\hline Q9 & 78 & 94 \\
\hline Q10 & 32 & 98 \\
\hline Q11 & 63 & 92 \\
\hline Q12 & 30 & 84 \\
\hline Q13 & 46 & 75 \\
\hline Q14 & 72 & 99 \\
\hline Q15 & 42 & 90 \\
\hline Average & 48.4 & 88.73 \\
\hline
\end{tabular}


impact of workshop is concerned. The study reveals that the number of attempts on MCQs irrespective of its correctness improved after the workshop was over than before it started. Simultaneously there was a significant rise in percentage of correct answers $(\mathrm{p}<0.001)$ across the participants in the post test as compared to pre-test which clearly depicts a strong positive impact of the workshop on the participants and such pre/post questionnaire clearly assisted in estimating such impression. Thus, administering pretests before lectures probably increased the attentiveness, curiosity, eagerness to listen to the lecture. An almost identical study involving pre and posttest module among pharmacy students revealed similar outcome ${ }^{11}$ as well as the study of Hartley $\mathrm{J}^{12}$ suggesting that pre-tests can be orienting and motivational.

In another study conducted by Jayachandra AL et al. also concluded similar findings as found in our study. ${ }^{13} \mathrm{It}$ can be presumed that the pretest questionnaire probably helped the participants to have an increased concentration and much effective learning from the workshop. Pretest probably helped the participants to focus better in the presentations during the workshop and post-test helped them to recall and retain the subject matter better similar to Dhawan et al and Nachiket et al. ${ }^{14,15}$ However, a study by Hill has observed that pretest does not have an assessable increase in the learning which had a contrasting outcome as compared toour study. ${ }^{16}$

Overall the pre/post questionnaire exhibited a good assessment and feedback tool particularly in workshop settings. Testing by MCQs questionnaire is an effective method to promote the retention of the knowledge gained during a particular session like didactic lectures and interactive sessions.

Few limitations of this study were the sample size was small, correctness of individual questions of the same participants was not assessed, assumption skill used by the participants in answering the MCQs correctly was not evaluated and finally fairness in answering the questions without any side talks was not ensured during this study.

\section{CONCLUSIONS}

Based on the above data and findings, it can be concluded that the response to post-test questionnaire in the form of MCQs reflected a clear improvement in performance as compared to pre-test result irrespective of the type of attempt made in answering such questions i.e. assumption and/or with confidence. The application of a pre/posttest questionnaire was observed to be a feasible tool to shape group specific education programs. Therefore pre/post questionnaire could be effective and reliable tools in meeting stipulated learning objectives.

\section{REFERENCES}

1. Miller VA, Reynolds WW, Ittenbach RF, Luce MF, Beauchamp TL and Nelson RM. Challenges in measuring a new construct: Perception of voluntariness for research and treatment decision making. J Empir Res Hum Res Ethics 2009; 4:21-31.

2. Kember D and Leung DY. Establishing the validity and reliability of course evaluation questionnaires. Assess Eval High Educ 2008; 33:341-353.

3. Wong KL, Ong SF and Kuek TY. Constructing a survey questionnaire to collect data on service quality of business academics. Eur J Soc Sci 2012; 29:209-221.

4. Barge GL. Pre- and Post-Testing with More Impact. Journal of Extension 2007; 45(6):103.

5. Jayachandran $\mathrm{AL}$ and Balaji J. Introduction of pre and post lecture multiple choice questions for second year undergraduate medical students in microbiology: a technique to assess knowledge acquired from the lecture. Int J Res Med Sci 2016; 4:575-578

6. Khan M and Aljarallah BA. Evaluation of modified essay question and $M C Q$ as a tool for assessing cognitive skills. Int J Health Sci 2011; 5(1):39-43.

7. Haynie WJ. Effects of take-home and in-class tests on delayed retention learning acquired via individualized, self-paced instructional texts. Journal of Industrial Teacher Education 1991; 28:52-63.

8. Rothman KJ, Greenland S and Lash TL. Modern Epidemiology. Philadelphia, USA: Lippincott William and Wilkins; 2008. p. 128-147.

9. Cooper DR and Schindler PS. Business Research Methods. 9 th ed. New York: McGraw-Hill; 2006.

10. Pedisic Z, Bennie JA, Timperio AF, Crawford DA, Dunstan DW, Bauman $A E$, et al. Workplace sitting breaks questionnaire (SITBRQ): An assessment of concurrent validity and test-retest reliability. BMC Public Health 2014; 14:1249.

11. Luetsch $\mathrm{K}$ and Burrows $\mathrm{J}$. Certainty rating in pre-and post-tests of study modules in an online clinical pharmacy course - A pilot study to evaluate teaching and learning BMC Medical Education (2016) 16:267.

12. Hartley J. The effect of pre-testing on post-test performance. Instr Sci 1973;2(2):193.

13. Jayachandran $\mathrm{AL}$ and Balaji J. Introduction of pre and post lecture multiple choice question for second year undergraduate medical students in microbiology: a technique to assess knowledge acquired from the lecture. Int J Res Med Sci 2016; 4:575-578

14. Dawane JS, Pandit VA, Dhande PP, Sahasrabudhe RA and Karandikar YSA. Comparative study of Different Teaching Methodologies used for developing understanding of Cardiac Pharmacology in Undergraduate Medical Students. IOSR Journal of Research \& Method in Education 2014:4(3):34-38.

15. Shankar $\mathrm{N}$ and Vallabhajosyula $\mathrm{R}$. Pre and post-lecture test scores for assessment of short-term effectiveness of didactic lectures in anatomy and as a predictor for performance in summative evaluation. South East Asian Journal of Medical Education 2012; 6(1):33-38.

16. Hill DA. Role of the pre-test in the progressive assessment of medical students. Aust N Z J Surg 1992; 62(9):743-746. 


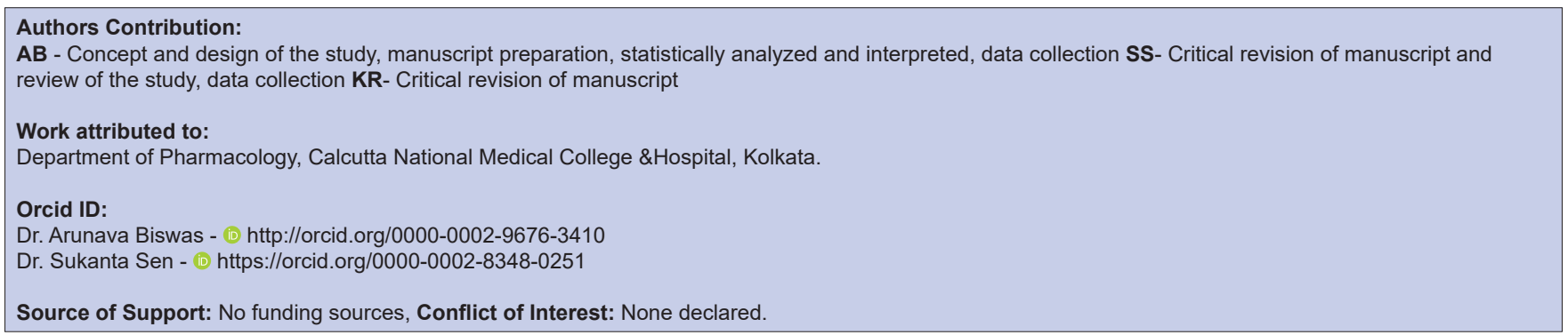

\section{ANNEXURE 1}

\section{A Pre-Conference Event of the 27th Annual Conference Indian Pharmacological Society, West Bengal IPSWBCon 2018}

\section{"Clinical Drug Research, Good Clinical Practice and Ethics" \\ CNMC, Kolkata-700014}

Tick the correct answers of the following questions: -

\section{PRE/POST TEST}

1) According to the principles of ICH GCP what is the most important consideration when conducting a clinical trial?
A. data accuracy
B. protection of trial subjects
C. Process adherence
D. Statistical quality checks

2) Which document created in 1964 forms the basis of ethical considerations in clinical research?
A. Declaration of Geneva
B. Declaration of Helsinki
C. Declaration of Belfast

3) What does ICH stand for?

A. International Convention on Homogenization

B. International Conference on Harmonization

C. International Conference on Homogenization

D. International Convention on Harmonization

4) What does ICH GCP state about the investigator or trial staff persuading subjects to take part in a trial? "Neither the investigator, nor the trial staff, should .................. a subject to participate or to continue to participate in a trial"
A. Persuade
B. Force
C. Coerce or unduly influence
D. Use inappropriate methods to influence

5) According to ICH GCP the investigator "should be qualified by.....”?
A. Training and experience
B. Education, training and experience
C. Education and experience
D. Education and training

6) According to ICH GCP where would you expect to find a section entitled" Summary of Data and Guidance for the Investigator"
A. Protocol
B. Clinical Study Report
C. Investigator Brochure
D. Investigator Agreement

7) The form 1572 "Statement of Investigator" is required by:
A. IRB
B. FDA
C. Sponsor
D. Investigator

8) Prior to subject's participation in the trial, the should be signed and personally dates by the subject or by the subject's LAR.
A. Protocol
B. Clinical Trial Agreement
C. IRB Approval Report
D. Written Informed Consent Form

9) Sponsor responsibilities include: -
A. Design study
B. Manage study
C. Finance study
D. Audit study
E. All above

10) A systematic and independent examination of trial-related activities and documents to determine whether the evaluated trial-related activities were conducted and the data were recorded, analysed, and accurately reported according to the protocol, sponsor's SOPs, GCP, and the applicable regulatory requirements.
A. Amendment
B. Site Evaluation
C. Audit
D. Inspection

12) What does IRB Stand for? 

A. Investigational Review Board
B. International Review Board
C. Institutional Review Board

13) Sponsor responsibilities include
A. Design study
B. Manage study
C. Finance study
D. Audit study
E. All the above

14) An individual or juridical or other body authorized under applicable law to consent, on behalf of a prospective subject, to the subject's participation in the clinical trial.
A. LAR (legally acceptable representative)
B. Subject
C. Trial Monitor
D. Investigator

15) An SAE (Serious Adverse Event) is any untoward medical occurrence that at any does:
A. Results in death
B. Is life-threatening
C. Requires inpatient hospitalization or prolongation of existing hospitalization
D. Results in persistent or significant disability/ incapacity
E. All the above 